\title{
An Automatic Recognizer for Iraqi License Plates Using ELMAN Neural Network
}

\author{
Abdulhussein Mohsin ${ }^{1}$, Abbas H. Hassin ${ }^{1,2}$, Iman Qais Abdul Jaleel ${ }^{1}$ \\ ${ }^{1}$ Computer Science Department, Science College, Basra University, Basra, Iraq; ${ }^{2}$ Atlantic County Institute of Technology, New Jer- \\ sey, USA. \\ Email:\{abdo60-2004, abhh2002, emankai\}@yahoo.com
}

Received October $11^{\text {th }}, 2010$; revised November $25^{\text {th }}, 2010$; accepted December $1^{\text {st }}, 2010$.

\begin{abstract}
License plate recognition system plays an important role in many applications. An automatic recognizer for Iraqi License Plates using ELMAN Neural network is proposed in this manuscript. The processing procedures are developed in several stages. Experimental results are reported in the end of the paper to illustrate the performance of the proposed method.
\end{abstract}

Keywords: Four Quadrant (4Q) Converter, Interlacing, Traction Systems, Power Quality Analysis

\section{Introduction}

Currently, there are more than half a billion vehicles on the worldwide [1]. All those vehicles have their identification number as a primary identifier. The vehicle identification number is actually a license number which means a legal license to participate in the public traffic. Also, should be fixed onto its body (at least at the backside).

In fact, the manual methods for dealing with more than half a billion vehicles are much difficult. Therefore, an automatic system - called license plate recognition (LPR) - is strongly needed. In general, LPR problem could be classified into two stages [2,3] plate image processing (including detection of plate from vehicle image, plate numbers and characters segmentation), and recognition of the isolated plate number.

In the field of isolated plate number recognition, a lot of conventional methods are used, for instance template matching [2,3], MPL neural networks [4,5], mathematical morphology [6], and image frequency analysis approach (such as DFT) [7].

In this paper, we present a new automatic and simple algorithm for recognizing Iraqi license plates. Basically, this algorithm consists of four main stages: 1) image acquisition. 2) preprocessing including cleaning of plate region from any noise by using median filter and removing unwanted lines by using morphological algorithms. 3) segmentation of cleaned plate into its characters and numbers by using horizontal and vertical pro- jection profiles. 4) recognition of the isolated plate number.

\subsection{Elements of LPR}

Normally, LPR consists of the following items:

- Camera, that takes the images of the vehicle (front or back side).

- Illumination, a controlled light that can bright up the plate, and allows day and night operation. In most cases illumination in Infra Red (IR) which is invisible to the driver.

- Computer, normally a PC, includes software and hardware requirements.

- Database, includes the vehicle information and recognition result.

\subsection{Typical Application of LPR}

There are several applications where automatic license plate recognition can be used. Those include the following:

- Parking: the plate number is used to automatically enter pre-paid members and calculate parking fee for non-members (by comparing the exit and entry times).

- Access Control: a gate automatically opens for authorized members in a secured area, thus replacing or assisting the security guard. The events are logged on a database and could be used to search the history of events. 
- Border Control: the car number is registered in the entry or exit to the country, and used to monitor the border crossings.

- Stolen cars: a list of stolen cars or unpaid fines is used to alert on a passing 'hot' cars. The 'black list' can be updated in real time and provide immediate alarm to the police force.

- Enforcement: the plate number is used to produce a violation fine on speed or red-light systems. The manual process of preparing a violation fine is replaced by an automated process which reduces the overhead and turnaround time. The fines can be viewed and paid on-line.

- Traffic control: the vehicles can be directed to different lanes according to their entry permits (such as in University complex projects).The system effectively reduces traffic congestions and the number of attendants.

- Marketing tool: his car plates may be used to compile a list of frequent visitors for marketing purposes, or to build a traffic profile (such as the frequency of entry verses the hour or day).

- Travel: a number of LPR units are installed in different locations in city routes and the passing vehicle plate numbers arematched between the points. The average speed and travel time between these points can be calculated and presented in order to monitor municipal traffic loads. Additionally, the average speed may be used to issue a speeding ticket.

- Airport Parking: in order to reduce ticket fraud or mistakes, the LPR unit is used to capture the plate number and image of the cars. The information may be used to calculate the parking time or provide a proof of parking in case of a lost ticket-a typical problem in airport parking which have relatively long (and expensive) parking durations.

\section{License Plate Image Detection}

In this stage, the license plate of vehicles are detected using digital camera infixed in anywhere of the street or in selected another place. When a vehicle passes in front of the camera, an image of vehicle are captured and stored for processing in next stage.

Unfortunately, captured image of Iraqi license plates has different colors (back-ground and foreground), different font styles for each plate in the same city, and noisy (see Figure 1), this makes the recognition of characters and numbers more difficult.

\section{Preprocessing}

In order to recognize the elements of the license plate accurately, image enhancements are required. Those en- hanceements include cleaning of plate region from any noise by using median filter and removing unwanted lines.

This stage starts by converting the color image into binary image. By thresholding the pixel values of 0 for all pixels in the input image with luminance less than threshold value and 1 for all other pixels (see Figure 2).

Median filter was used to remove significant noise then Sobel operator has been used, which is composed of two $3 \times 3$ masks, to determine the edge of the license plate. The plate may contains hard noise that the median filter couldn't remove because it is so enormously large, for that reason we calculate the number of columns, number of rows and the rate between them for each character found in the filtered binary image. By thresholding, we can determine whether the character stays or to be removed from the license plate.

Median filter was used to remove significant noise

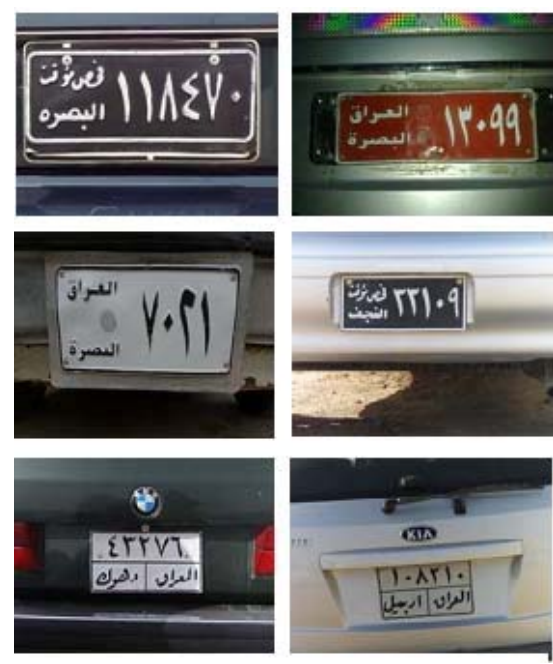

Figure 1. Samples of Iraqi license plate.

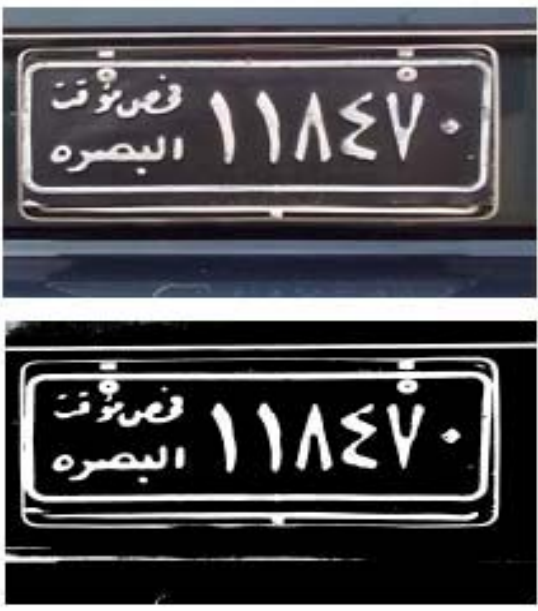

Figure 2. Captured and binary images. 
then Sobel operator has been used, which is composed of two $3 \times 3$ masks, to determine the edge of the license plate. The plate may contains hard noise that the median filter couldn't remove because it is so enormously large, for that reason we calculate the number of columns, number of rows and the rate between them for each character found in the filtered binary image. By thresholding, we can determine whether the character stays or to be removed from the license plate.

\section{Segmentation}

Segmentation is an essential step in any practical LPR system. There are many reasons that make the segmentation task a difficult one, such as image noise, plate frame rivet, and illumination variance. Before starting the segmentation stage we determine whether the license plate is written horizontally or vertically as shown in Figure 3.

After determining the plate style, an algorithm has been applied. If the plate style is vertical we determine the values of the pixels in each column of the entered plate, and then count the number of pixels of value 1 in each column to determine the following:

1) The beginning and the end of the segment that contains the numbers and characters by removing the sum of columns with number of pixels $<\mathrm{T}$ (experimentally $\mathrm{T}=10)$.

2) Edge that separates the numbers from characters by determining the column with number of pixels less than its neighbors from left and right. Figure 4 shows this process.

If the plate style is horizontal we use the vertical search to find the sum of pixels of the plate characters. To determine the edge between the plate numbers and

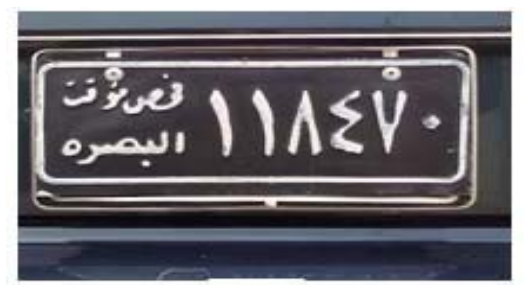

(a)

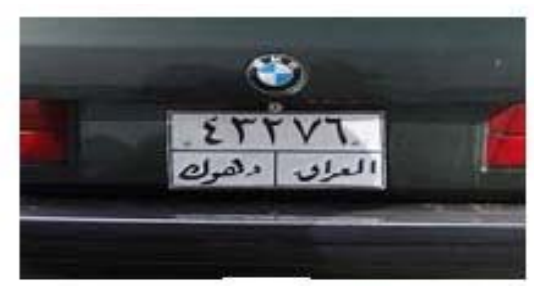

(b)

Figure 3. The style of Iraqi license plate. (a) vertical style; (b) horizontal style.

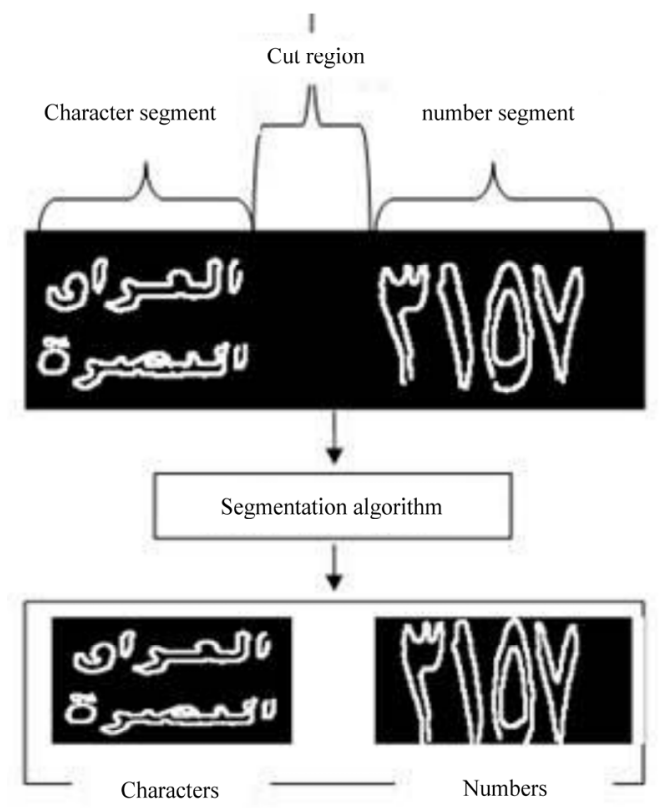

Figure 4. Example of segmentation algorithm.

other character, we use the smaller value between any two maximum values.

\section{Recognition}

For accurate discrimination template should be retained each number from 0 to 9 as well as the template for each word represents the Iraqi provinces, such as Basra, Najaf, and Baghdad as well as word that represents the name of the state, i.e., Iraq and other words that appear on the plate. In order to minimize the data used as inputs to the system of discrimination, we use the longest section of the text in any word in the word "إلبصرة" that

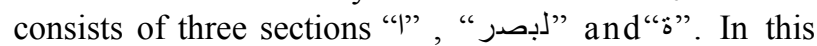
case, we use the section " لبصر" since the other province names have no such section.

Two of Elman neural networks (ENN) topologies are used in recognition stage. One for recognizing 10 numbers (0.9), another for recognizing 9 province names. A function is developed in designing this ENN has three layers consisting of input layer, hidden layer and output layer. In the ENNs there will be 256, 20, 10 neurons for input, hid- den, and output layers respectively as shown in Figure 5 and Figure 6.

The value of element of each targets are all zero except one element on specific position which represent the number or text segment. For example, the first element is 1 in number's target, represent 0 . To recognize, output should be processed first by converted the highest value to be 1 and other will be 0 .Then, the location of element which has value 1 will be founded and the result will represent number or governorate name. 


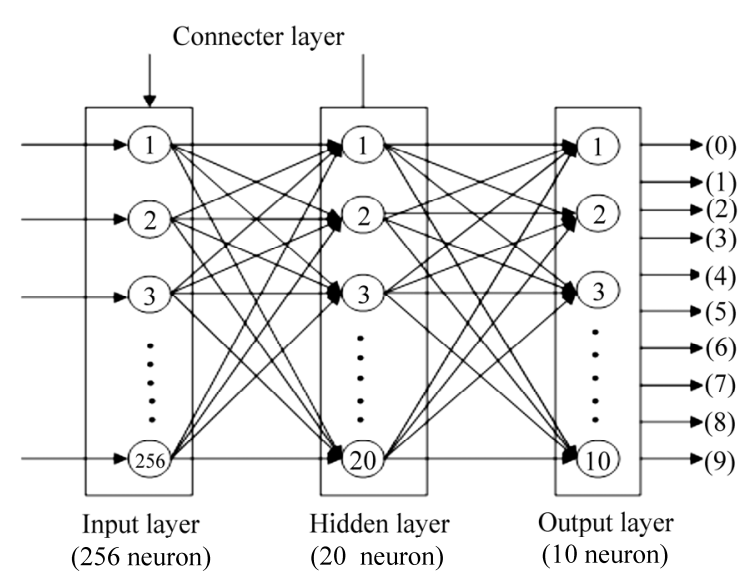

Figure 5. Iman neural network of recognize numbers in Iraqi license plate.

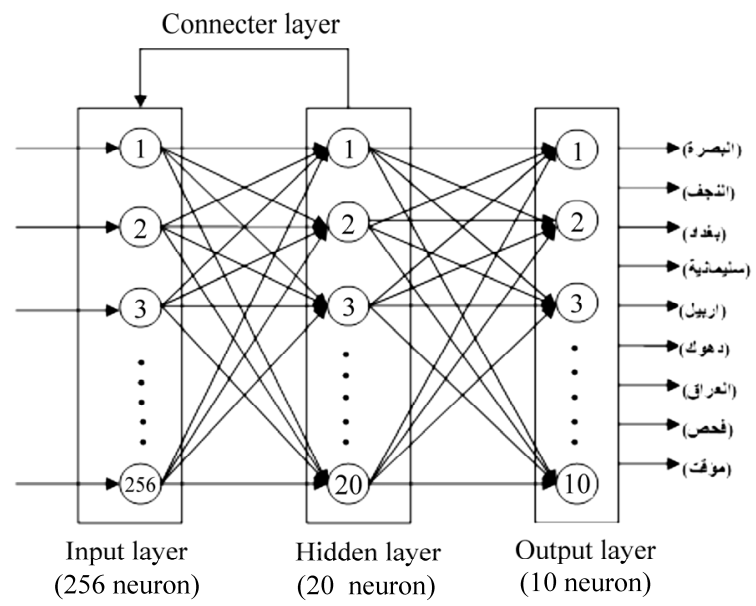

Figure 6. Elman neural network of recognize characters in Iraqi license plate.

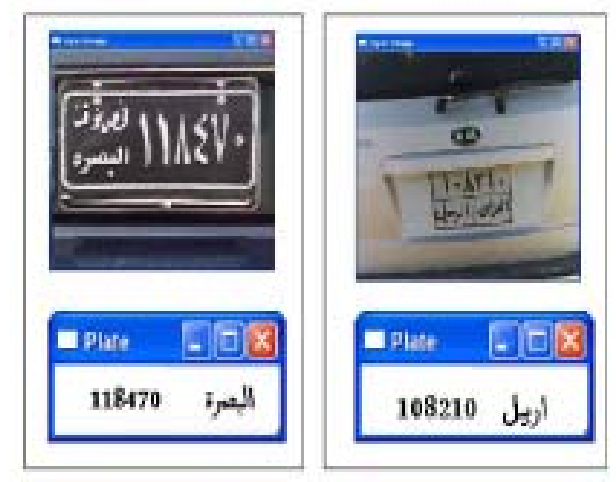

Figure 7. Results of proposed algorithm.
Table 1. Experimental results.

\begin{tabular}{lcc}
\hline Applied Stage & Correct Detection & Accuracy (\%) \\
\hline Segmentation & $18 / 21$ & $85 \%$ \\
Recognition & $18 / 21$ & $76 \%$ \\
\hline
\end{tabular}

\section{Conclusions}

In this paper, we introduced a new algorithm for Iraqi license plate number recognition.

The test images were taken under various illuminations, size and types of license plate conditions. The experimental results for the LPR system were performed under different illuminations, different distances and various types of license plate. The experimental results (see Figure 7), show that the algorithm achieved about $85 \%$ correct segmentation and $76 \%$ correct recognition for 21 samples as shown in Table 1.

\section{REFERENCES}

[1] A. Amin, "Off-Line Arabic Character Recognition-A Survey," Proceedings of the 4th International Conference on Document Analysis and Recognition; Ulm, Germany, 18-20 August 1997, pp. 596-599. doi: 10.1109/ICDAR.1997.620572

[2] W. Al Faqheri and S. Masho-hor, "A Real-Time Malaysian Automatic License Plate Recognition (M-ALPR) Using Hybrid Fuzzy," International Journal of Computer Science and Network Security, Vol. 9, No. 2, 2009, pp. 333-340.

[3] M. Kumar, "A Real-Time Vehicle License Plate Recognition (LPR) System," Master's Thesis, Thapar University, Patiala, 2009.

[4] N. K. Babu and K. Nallape-rumal, "An Efficient Geometric Feature Based License Plate Localization and Recognition," International Journal of Imaging Science and Engineering, Vol. 2, No. 2, 2008, pp. 189-194.

[5] A. Akoum, B. Daya and P. Chauvet, "Two Neural Networks for License Number Plates Recog- nition," Journal of Theoretical and Applied Information Technology, Vol. 12, No. 1, 2009, pp. 25-32.

[6] S. H. Kasaei, S. M. Kasaei and S. A. Kasaei, "New Morphology Based Method for Robust Iranian Car Plate Detection and Recognition," International Journal of Computer Theory and Engineering, Vol. 2, No. 2, 2010, pp. 264-268.

[7] S. Kim, S. Oh and M. Kang, "Mathematical Image Processing for Automatic Number Plate Recognition System," Journal of the Korean Society for Industrial and Applied Mathematics, Vol. 14, No. 1, 2010, pp. 5766. 